\title{
Discours, plurilinguisme et construction des savoirs dans les MOOC et les formations entièrement ou partiellement à distance Présentation du numéro
}

\section{Discourse, plurilinguism and knowledge construction in MOOC and other forms of distance education}

\section{Discursos, multilingüismo y construcción de conocimiento en los MOOC y en formaciones parcial o totalmente a distancia}

https://doi.org/10.52358/mm.vi7.259

RESPONSABLES DU NUMÉRO

Mariana Fonseca Favre, maître-assistante

Université de Genève, Suisse

mariana.fonseca@unige.ch

Claire Peltier, professeure adjointe

Université Laval, Canada

claire.peltier@fse.ulaval.ca

Baptiste Campion, chargé de cours

Institut des Hautes Études des Communications Sociales (IHECS), Belgique

baptiste.campion@galilee.be 
RÉSUMÉ

Les $M O O C$ et autres dispositifs de formation entièrement ou partiellement à distance ont souvent recours, notamment pour des raisons d'accessibilité, à la langue anglaise et à des formats médiatiques assez standardisés (tel le face camera). Toutefois, les implications des choix d'écriture posés lors de la conception de ces productions éducatives restent insuffisamment interrogées. Cet éditorial cherche à mettre en perspective les questions transversales soulevées par ces choix de réalisation, leurs causes et leurs conséquences possibles. II argumente de la nécessité de développer des recherches interdisciplinaires sur ces dispositifs de formation à distance reposant en grande partie sur la vidéo, en remettant en question, de manière systématique, la manière dont s'articulent en leur sein les composantes linguistique, discursive et médiatique, dans des contextes institutionnels et techniques généralement structurants. II conclut en présentant les contributions de ce numéro consacré au rôle des langues et des discours dans la construction des savoirs dans les $M O O C$ et autres dispositifs de formation à distance.

Mots-clés : $M O O C$, formation à distance, plurilinguisme, langages, discours

\begin{abstract}
MOOC and other entirely or partially distance learning devices often use English and fairly standard media formats, notably due to accessibility concerns. However, the implications of the choices made in the design of these educational productions remain insufficiently explored. This editorial seeks to put into perspective the cross-cutting issues raised by these choices, while identifying their determinants and consequences. We argue for the need to develop interdisciplinary research on these distance learning devices mainly based on videos. To do so, we systematically question how linguistic, discursive and media components are articulated in institutional and technical structuring contexts. We conclude by presenting the contributions in this issue, which is devoted to the role of languages and discourses in the construction of knowledge in $\mathrm{MOOC}$ and other distance learning devices.
\end{abstract}

Keywords: MOOC, distance learning, plurilingualism, languages, discourses

\title{
RESUMEN
}

Los MOOC y otras modalidades de formación total o parcialmente a distancia suelen utilizar el inglés, especialmente por razones de accesibilidad, así como formatos mediáticos bastante estandarizados. Sin embargo, las implicaciones de las selecciones de escritura realizadas en el diseño de tales producciones educativas siguen siendo insuficientemente exploradas. Este editorial trata de poner en perspectiva las cuestiones transversales que plantean dichas opciones de producción, sus causas y sus posibles consecuencias. En él, se defiende la necesidad de desarrollar una investigación interdisciplinar sobre estas modalidades de formación a distancia basadas en buena medida en el vídeo, cuestionando sistemáticamente el modo en que se articulan en las mismas los componentes lingüísticos, discursivos y mediáticos, en contextos institucionales y técnicos generalmente estructurantes. El editorial 
se cierra con la presentación de las aportaciones de este número dedicado al papel de las lenguas y de los discursos en la construcción del conocimiento en los MOOC y otras modalidades de formación a distancia.

Palabras clave: MOOC, educación a distancia, plurilingüismo, idiomas, discursos

\section{Les MOOC, objets linguistiques et langagiers}

Depuis quelques années, les cours en ligne ouverts et massifs (MOOC, ou CLOM en français) contribuent à une large diffusion des savoirs, principalement à travers la mise à disposition de contenus diversifiés destinés à un public d'adultes intéressés par des formations courtes, parfois diplômantes, et aux modalités de suivi flexibles. Initialement portés par de grandes universités occidentales (surtout aux États-Unis et en Europe), les $M O O C$ font aujourd'hui l'objet d'une offre étendue et hétérogène, tant du point de vue des thématiques abordées que du modèle économique sous-jacent (Cisel, 2018). Deux aspects semblent pourtant particulièrement stables et récurrents dans ce contexte : le recours massif à la langue anglaise et la prédominance, à travers la majorité des capsules vidéo produites, d'une forme de magistralité discursive caractéristique d'une approche pédagogique transmissive.

Souvent justifié par une recherche d'universalité des MOOC, elle-même motivée par des politiques institutionnelles et/ou de diffusion, le recours à une langue hégémonique n'est pas sans conséquence pour les processus de construction et de contextualisation des savoirs. Les travaux sur le plurilinguisme, notamment dans l'enseignement supérieur, ont montré que l'utilisation massive de l'anglais repose souvent sur une représentation des langues mettant en avant de manière parfois un peu simpliste sa fonction de communication et sous-exploitant leur potentiel pour la construction des connaissances (Berthoud et Gajo, 2020). Par ailleurs, I'homogénéisation non seulement des langues, mais également des formats discursifs et pédagogiques, induite par les grandes plateformes, tend vers des modèles universels de diffusion des connaissances (Altbach, 2014), gommant l'ancrage culturel des savoirs (Dillon, Wang et Tearle, 2007), pourtant présent tant du côté de la conception que de la réception (Fonseca et Gajo, 2020, 2021). Malgré cette tendance à la standardisation, certains travaux commencent à mettre en évidence, à partir de perspectives diverses, l'importance de prendre en compte la diversité linguistique et culturelle dans les MOOC (Bayeck et Choi, 2018; Kerr, Merciai et Eradze, 2018; Nkuyubwatsi, 2014; Phan, 2018; Stratton et Grace, 2016). Si ces contributions constituent des avancées importantes, elles travaillent généralement dans une perspective d'ouverture à la diversité, s'intéressant rarement au rôle du plurilinguisme dans la construction des savoirs.

Du point de vue discursif et pédagogique, les MOOC présentent certaines particularités qu'il semble intéressant d'aborder sous l'angle linguistique et communicationnel. Depuis plusieurs décennies, anthropologues, psychologues et psycholinguistes ont en effet montré l'importance du langage dans le développement cognitif humain. Au-delà de son rôle structurant, le langage - et en particulier le langage verbal - est porteur d'un ensemble de représentations du monde, lesquelles diffèrent d'une langue à l'autre. En effet, en tant que système de signes arbitraires, une langue constitue un cadre d'opérations mentales et l'expression singulière d'une culture et de valeurs, enfin une façon de voir le monde. Du point de vue cognitif, Denis (1989) relevait la capacité qu'ont les mots à produire des images mentales distinctes. 
De leur côté, Fauconnier et Turner (2002) ont souligné le rôle des métaphores langagières dans l'élaboration conceptuelle. Enfin, les travaux de Gajo (voir, par exemple, Gajo, 2007) ont mis en évidence que le passage par une deuxième langue (L2), en permettant une deuxième médiation (une « remédiation »), contribue souvent à une meilleure intégration des savoirs linguistiques et disciplinaires. Par ces quelques exemples, on observe que, contrairement à la représentation courante, les langues ne sont pas des véhicules transparents (voir Cassin, 2004), mais peuvent jouer un rôle non négligeable dans la construction des savoirs.

Dans le même ordre d'idées, si l'on se réfère aux travaux menés en sémiologie et en sémiopragmatique, les capsules vidéo produites à des fins éducatives dans les $M O O C$ ou tout autre dispositif de formation entièrement ou partiellement à distance peuvent être appréhendées comme des langages articulant plusieurs registres sémiotiques (audio-scripto-visuel selon la terminologie de Cloutier, 1973) susceptibles de produire des effets différenciés sur les apprenants (Meunier et Peraya, 1993/2010; Peraya, 1999). Plusieurs études récentes (Peltier et Campion, 2017; Peltier et Campion, 2018; Campion, Peltier et Peraya, 2019) ont souligné que la nature discursive des capsules vidéo élaborées pour les $M O O C$ fait la part belle au discours magistral et à une vision diffusionnelle et transmissive de l'apprentissage médiatisé. Dans cette perspective, la dimension relationnelle, pourtant intrinsèquement constitutive de tout langage et de tout apprentissage, est le plus souvent occultée et sous-exploitée sur le plan verbal.

\section{Une problématisation nécessaire}

Questionner les $M O O C$ et autres dispositifs de formation intégralement ou partiellement à distance dans leurs aspects linguistiques, discursifs et communicationnels ne s'impose néanmoins pas comme une évidence, et ce, pour plusieurs raisons. D'une part, ces dimensions des capsules vidéo sont étudiées par des chercheurs appartenant à des champs disciplinaires proches, comme la linguistique et la sémiotique, ou encore les sciences de l'éducation et de l'information, mais qui n'abordent que rarement cet objet récent et complexe de manière coordonnée. D'autre part, le développement de ces dispositifs de médiation des savoirs se fait, on le verra, de manière parfois assez empirique, les acteurs développant avant tout des solutions par expérimentation directe, voire par essais et erreurs, le travail de conceptualisation ne se faisant souvent que dans un second temps, une fois que les pratiques se stabilisent et s'institutionnalisent. Une des ambitions de ce numéro thématique est de proposer des axes de réflexion forts propices à l'articulation disciplinaire et à la réflexivité propre à interroger des pratiques parfois déjà bien ancrées dans les établissement d'enseignement universitaire. Un tel projet impose, cependant, de préalablement problématiser cet objet en identifiant brièvement les questions transversales à la médiation des savoirs au sein des $M O O C$ et de leur production.

\section{Médiation numérique et accès aux savoirs}

Quand on s'intéresse à l'enseignement et à l'apprentissage à distance, on ne peut que faire le constat de la centralité qu'y occupent désormais les capsules vidéo utilisées à des fins de médiation des savoirs, que ce soit sur des plateformes grand public (comme YouTube) ou encore spécialisées dans les MOOC (comme Coursera, FUN, OpenClassroom, MyMOOC, etc.) (voir Cisel et Bruillard, 2012). La place prise par la vidéo dans ce dispositif est devenue si importante que certains auteurs la considèrent même comme une nouvelle forme de manuel scolaire pour l'université (Young, 2013). 
Cette évolution n'est pas sans conséquence sur la manière de construire et de scénariser ces dispositifs de formation à distance, pour différentes raisons. D'une part, la vidéo, en permettant d'atteindre un public plus large, constitue un vecteur puissant de massification de l'enseignement. D'autre part, elle tend à imposer, comme le notaient déjà Cisel et Bruillard (2012) cités plus haut, des formats courts, à même de répondre à des pratiques de consultation fragmentées, amenant ainsi à ce que Michaël Bourgatte et Daniel Jacobi (2019) identifient comme une forme "d'hypersegmentation du savoir ». Cette nécessité de formatage les amène au concept de "vidéographisation », qu'ils définissent comme un " principe de médiation du savoir passant par la réalisation de vidéos » la plupart du temps destinées à une diffusion sur Internet (Bourgatte et Jacobi, 2019). Ainsi, pour les concepteurs, produire des capsules vidéo présuppose un important travail d'écriture médiatique adaptée au contexte numérique, qui passe par différents procédés de médiation - pas toujours conscients et donc variablement problématisés - visant à réduire la distance entre les savoirs savants et les savoirs ordinaires.

Nous pouvons tenter de décrire cette écriture médiatique à travers l'identification d'au moins trois composantes, qu'il ne s'agit pas simplement de juxtaposer, mais dont il faut également penser l'intégration de sorte à prendre pleinement en compte la multimodalité numérique dans l'accès aux savoirs. Ces composantes sont :

- la composante linguistique : choix de la ou des langues de la capsule vidéo et/ou des variétés de langue, prise en compte des registres langagiers, éventuellement réflexion sur la mise à disposition d'un dispositif de sous-titrage;

- la composante discursive et les modèles de magistralité qui en découlent, à mettre en relation avec le format de la séquence : exposé face camera, témoignage, entretien, table ronde sont parmi les formats les plus couramment employés dans les capsules vidéo des MOOC et mobilisent des types de discours spécifiques - discours théorique, narration, dialogue, etc. Si on suit (Jacquinot, 1977/2012), on pourrait se demander quels seraient les formats à adopter afin de sortir d'une stricte « épistémologie de la transmission »;

- la composante communicationnelle ou "médiatique »: intégration des aspects propres à l'écriture audiovisuelle, comme la disposition des interlocuteurs face à la caméra, le choix du lieu, l'échelle des plans, etc. qui contribuent à construire une relation de proximité avec l'apprenant. À cela s'ajoutent les incrustations d'illustrations, schémas, mots-clés, qui sont autant de manières de didactiser le savoir. Contrairement aux précédentes, du ressort des concepteurs, cette composante " médiatique » dépend potentiellement d'un nombre d'acteurs plus important, car elle est construite à la croisée d'un savoir disciplinaire détenu par les enseignants-chercheurs, mais également de l'équipe pédagogique, didactique et technique de production.

De manière transversale à ces trois composantes se trouve la dimension culturelle, qui peut être comprise à deux niveaux, comme culture nationale, mais aussi comme culture disciplinaire (voir Fonseca et Gajo, 2020). Les choix opérés par les concepteurs et équipes sont ainsi marqués par le contexte dans lequel ils se trouvent (Bayeck et Choi, 2018) - on n'envisage pas nécessairement les dimensions citées plus haut de la même manière à Genève, à Bruxelles ou à Québec - et par la discipline de référence (voir, par exemple, Fonseca et Gajo, 2021; Bourgatte et Jacobi, 2019) - une capsule qui développe un sujet de droit n'opère pas les mêmes choix d'écriture médiatique qu'une capsule qui traite un sujet de médecine. Par ailleurs, à l'intérieur même d'un domaine disciplinaire, le choix de la ou des langues et/ou des registres, 
par exemple, peut varier selon le sujet traité (voir l'entretien avec Laurent Turcot ici même'). Rappelons, par ailleurs, que la dimension culturelle ne concerne pas uniquement les pratiques des concepteurs, mais également celles des apprenants/usagers (Loizzo et Ertmer, 2016). Cette dimension renvoie à la tension entre les processus de contextualisation et d'internationalisation qui anime les $M O O C$ et qui reste encore insuffisamment interrogée (Fonseca et Gajo 2021; Gajo, Fonseca et Steffen ici même²).

II semble ainsi nécessaire de questionner ce rapport au plurilinguisme et aux langages (compris ici dans le sens de l'articulation de plusieurs registres sémiotiques dans une capsule vidéo) dans l'ensemble des contraintes techniques et institutionnelles pesant sur la conception de ces dispositifs médiatisés. D'un côté, la mise en forme technique nécessaire définit un ensemble de possibles avec lequel les intentions pédagogiques doivent être « négociées ». Par exemple, tout n'est pas aisément facile à montrer à l'image, ou encore le recours au sous-titrage dans une perspective plurilingue impose des choix de réalisation qui vont, à leur tour, influer sur la posture des apprenants/usagers. D'un autre côté, les politiques institutionnelles contribuent à cadrer parfois fortement la manière dont sont pensées, réalisées et produites les capsules vidéo, comme nous l'avons déjà mentionné. Cela est d'autant plus vrai que ces contextes institutionnels sont parfois très différents les uns des autres, et font par ailleurs l'objet d'évolutions (voir $p$. ex. Jaillet, 2014), allant de situations où les enseignants sont contraints de « bricoler » avec les moyens du bord à de véritables politiques institutionnelles dotées de moyens conséquents. À cela s'ajoute le fait que ces capsules sont, ensuite, mises en ligne sur des plateformes qui ont leurs propres logiques économiques ainsi que des modèles de mise en activité des apprenants et d'accompagnement des contributeurs (Bullich, 2018), allant souvent dans le sens d'une «internationalisation » (et parfois marchandisation) des savoirs (Moeglin, 1998). Pour cette raison, il serait également utile de s'interroger sur les conséquences possibles de ces politiques institutionnelles, voire entrepreneuriales, sur l'homogénéisation des savoirs, leurs modes de médiation et les pratiques présidant à leur " consommation », mais cela sortirait de notre propos.

Enfin, on notera que ces expériences interrogent également la question du statut de ces productions dans le champ universitaire et leur reconnaissance par les établissements d'enseignement. On rencontre en effet des discours potentiellement contradictoires en la matière, entre un espoir de (re)valorisation de la figure du pédagogue par l'entremise de l'investissement dans ces dispositifs innovants et sources de prestige pour les universités, et la faible reconnaissance par les pairs de ces productions et du temps que les universitaires peuvent y consacrer.

\footnotetext{
${ }^{1}$ https://doi.org/10.52358/mm.vi7.214

${ }^{2}$ https://doi.org/10.52358/mm.vi7.223
} 


\section{Présentation du numéro thématique}

Les contributions constituant ce dossier rendent compte d'expériences menées dans des contextes différents, et prennent des formes variées : de l'article de recherche " classique " à l'entretien avec un praticien, en passant par des comptes-rendus de travaux d'étudiants et un témoignage sur les expériences menées dans un contexte institutionnel particulier. Cette diversité est une richesse de ce numéro et une volonté de ses coordonnatrices et coordonnateur, soucieux de montrer, par-delà les contextes institutionnels et disciplinaires, que chaque expérience apporte à sa manière des éléments de réponse aux questions éminemment transversales que nous venons de présenter.

\section{Articles de recherche}

L'article d'Annick Rivens Mompean et Sophie Babault fournit des pistes intéressantes pour questionner l'articulation des logiques linguistiques, langagières, pédagogiques et techniques nécessairement à l'œuvre dans un $M O O C$, dont l'articulation est par essence complexe. Partant de leur expérience comme expertes dans la mise en place d'un $M O O C$ en didactique des langues et de l'observation de $M O O C$ déjà existants, leur contribution questionne les paradoxes inhérents à la création d'un $M O O C$ en didactique des langues, dont différents choix pédagogiques sont en réalité guidés par des contraintes techniques propres à cet objet. Les auteures montrent que ces dispositifs d'apprentissage imposent de penser les tensions et les régulations possibles entre quatre pôles interconnectés présents dans tout $M O O C$ : les conceptions de l'apprentissage sur lesquelles s'appuient les concepteurs, le public visé et ses caractéristiques, les choix techniques de mise en œuvre du dispositif et, enfin, le cadre institutionnel dans lequel le MOOC prend place. Ces tensions appellent des régulations qu'elles proposent de modéliser (modèle APTI), inscrites dans deux temporalités différentes: d'une part celle de la conception des $M O O C$, à travers le poids relatif donné aux différents pôles, et d'autre part celle de la mise en œuvre du MOOC proprement dite, à travers une attention particulière portée aux discours d'accompagnement.

En partant du principe que le dispositif détermine la transposition didactique (Chevellard et Joshua, 1991), Audrey Thonard 4 interroge, à partir d'exemples concrets, l'effet d'un $M O O C$ destiné à de futurs enseignants de FLE sur la médiation (cognitive, relationnelle et langagière, Coste et Cavalli, 2020) des savoirs. Dans ses analyses, l'auteure montre que les dimensions relationnelle et cognitive sont omniprésentes sur le plan verbal (à travers, notamment, le recours, par l'enseignant, à différents genres de discours, à une posture énonciative polyphonique) et non verbal (gestes, mimiques) et contribuent à la construction des connaissances, malgré la distance. Si l'aspect « ouvert » et « en ligne » du MOOC n'est pas un écueil pour la construction de la relation pédagogique entre enseignant et enseigné, cette contribution montre que cela n'est pas le cas pour la « massivité » qui complexifie l'accompagnement des participants et demande l'adoption d'autres formules comme les SPOC (Small Private Online Course).

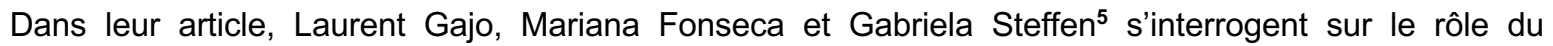
plurilinguisme dans les $M O O C$ et, en particulier, le mécanisme d'alternance codique, bien décrit dans les études du biplurilinguisme, mais encore peu appliqué au cas des MOOC. Les auteurs expliquent que ce mécanisme peut intervenir à différents niveaux macro (choix de la langue pour telle capsule vidéo ou tel

\footnotetext{
${ }^{3}$ https://doi.org/10.52358/mm.vi7.217

${ }^{4}$ https://doi.org/10.52358/mm.vi7.209

${ }^{5}$ https://doi.org/10.52358/mm.vi7.223
} 
$M O O C$ ), micro (changement de langue plus ou moins spontané en cours d'énoncé) et méso (recours didactisé à deux ou plusieurs langues dans une même capsule ou leçon) - et concerne notamment la place et l'usage du sous-titrage dans ce dispositif. Au moyen d'extraits de trois $M O O C$ issus de différentes disciplines, de déclarations des concepteurs et de témoignages des usagers, les auteurs décrivent le potentiel du plurilinguisme non seulement pour la communication des savoirs dans les MOOC, mais également pour leur problématisation. Par ce biais, cette contribution met en évidence la tension entre l'universalisation - choix d'une langue unique pour la diffusion des savoirs - et la contextualisation - prise en compte de la diversité linguistique et culturelle dans la fabrication des savoirs - qui anime ce dispositif.

\section{Travaux étudiants}

La contribution de Teurra Villattí présente et analyse une expérience de réalisation d'une capsule vidéo plurilingue pour la diffusion de sa recherche doctorale auprès d'un public de non-spécialistes dans le cadre d'un événement promu par une université brésilienne. À partir d'une analyse qualitative et interprétative de sa propre production, l'auteure suggère comment la mobilisation du plurilinguisme comme stratégie discursive peut contribuer à la valorisation et à transmission du savoir scientifique.

Dans sa contribution, Carole Cattin ${ }^{7}$ cherche à identifier quelques enjeux pédagogiques du passage de deux enseignements universitaires présentiels à un MOOC. Pour ce faire, elle étudie les choix et contraintes qui ont pesé sur la conception d'un MOOC, qui allie plurilinguisme (diversité des langues) et variation (diversité au sein d'une même langue). Ce texte souligne en particulier la manière dont ce dispositif amène les concepteurs à produire un travail d'écriture médiatique numérique, qui touche aux dimensions linguistiques, discursives et communicationnelles exposées plus haut (même si ces notions ne sont pas décrites en ces termes dans la contribution). Ce travail peut compliquer et complexifier la scénarisation du dispositif en raison de la tension émergeant entre un souhait d'accessibilité large et les niveaux de maîtrise linguistique exigée des usagers pour saisir toutes les implications de ces choix.

\section{Témoignages et entretiens}

Dans un long entretien avec l'historien et vidéaste Laurent Turcot, Baptiste Campion et Claire Peltier interrogent la construction de ces dispositifs d'apprentissage non sous l'angle de la langue, mais de la construction discursive et communicationnelle des capsules vidéo telles qu'elles sont utilisées dans de nombreux MOOC, dispositifs de formation à distance ou de vulgarisation. Parlant de son expérience de vulgarisateur des savoirs historiques sur la plateforme YouTube, Laurent Turcot s'attarde sur les questions d'écriture au sens large du terme : une capsule vidéo articule un texte dont il met en avant les contraintes d'efficacité et de fluidité, et une écriture audiovisuelle à travers les choix de cadrage, la variation des plans et le rythme des illustrations, le tout au service d'une économie de l'attention particulièrement importante sur ce type de plateforme. Ce faisant, cet entretien montre également les ressources pratiques et techniques mobilisées pour construire de telles capsules pédagogiques ou de vulgarisation, souvent mal maîtrisées par les universitaires à l'origine de ces dispositifs, et pas toujours reconnues dans le champ universitaire.

\footnotetext{
${ }^{6} \mathrm{https://doi.org/10.52358/ \textrm {mm } . v i 7 . 2 0 6}$

${ }^{7}$ https://doi.org/10.52358/mm.vi7.215

${ }^{8}$ https://doi.org/10.52358/mm.vi7.214
} 
Cette question des compétences des enseignants-chercheurs articulée à la recherche de dispositifs pédagogiques performants basés sur des capsules vidéo avec leurs spécificités langagières est au cœur de la contribution de Stéphanie Chauveau'. Celle-ci rend compte de l'expérience de Michaël Bourgatte et Laurent Tessier, enseignants-chercheurs à I'Institut Catholique de Paris et concepteurs d'un projet de laboratoire universitaire des innovations pédagogiques. Ceux-ci ont en effet intégré la vidéo dans leurs projets dès 2014 et témoignent d'une évolution pédagogique et technique structurée à la fois par une réflexion sur les dispositifs pédagogiques et ce qu'on pourrait considérer comme des hasards, comme les compétences en vidéo d'un membre de l'équipe ou les partenariats qui permettent de développer ces projets. Par l'intermédiaire de Stéphanie Chauveau, Michaël Bourgatte et Laurent Tessier montrent comment cela les a amenés à s'éloigner progressivement du "modèle " de l'enseignant face camera soutenant une magistralité transmissive visible dans un grand nombre de $M O O C$ au profit de la mise en place de dispositifs visant à la collaboration entre enseignants et apprenant à travers la vidéo, par le biais du concept de vidéographisation que nous avons évoqué plus tôt.

\section{Liste de références}

Altbach, P. (2014). MOOCs as neocolonialism: who controls knowledge? International Higher Education, 75, 5-7. https://doi.org/10.6017/ihe.2014.75.5426

Bayeck, R.Y. et Choi, J. (2018). The influence of National Culture on Educational Videos: The Case of MOOCs. International Review of Research in Open and Distributed Learning, 19(1), 186-201. https://doi.org/10.19173/irrodl.v19i1.2729

Berthoud, A.-C. et Gajo, L. (2020). The Multilingual Challenge for the Construction and Transmission of Scientific Knowledge. Amsterdam/Philadelphia : John Benjamins.

Bourgatte, M. et Jacobi, D. (2019). Les médiatisations visuelles des savoirs scientifiques. Dans B. Lafon (dir.), Médias et médiatisation (p. 241-271). Presses universitaires de Grenoble. https://doi.org/10.3917/pug.lafon.2019.01.0241

Bullich, V. (2018). La " plateformisation» de la formation. Distances et médiations des savoirs. Distance and Mediation of Knowledge, 2018(21), article 21. https://doi.org/10.4000/dms.2096

Campion, B., Peltier, C. et Peraya, D. (2019). Analyse communicationnelle de vidéos de MOOCs : quelle magistralité les choix discursifs construisent-ils? Éducation et formation, e-313. http://revueeducationformation.be/include/download.php?idRevue=33\&idRes $=354$

Cassin, B. (dir.) (2004). Vocabulaire européen des philosophies. Dictionnaire des intraduisibles. Éditions du Seuil/Dictionnaires Le Robert.

Cisel, M., et Bruillard, E. (2012). Chronique des MOOC. Sciences et Technologies de l'Information et de la Communication pour l'Éducation et la Formation, 19(1), 49-73. https://doi.org/10.3406/stice.2012.1035

Coste, D. et Cavalli, M. (2020). Des traits constitutifs de toute médiation? Dans De Goia M. et Marcon M. (dir.). L'essentiel de la médiation. Le regard des sciences humaines et sociales (p. 261.288). Peter Lang.

Chevellard, Y. et Joshua, M.-A. (1991). La transposition didactique: du savoir savant au savoir enseigné. Grenoble : La Pensée sauvage.

${ }^{9}$ https://doi.org/10.52358/mm.vi7.208 
Cisel, M. (2018). Les MOOC français à l'épreuve de la viabilité économique. Revue française des sciences de l'information et de la communication, 12. https://journals.openedition.org/rfsic/3355

Cloutier, J. (1973). La communication audio-scripto-visuelle. Communication \& langages, 19, 75-92. https://www.persee.fr/doc/colan 0336-1500 1973 num 1914033

Denis, M. (1989). Les images mentales. Paris : Presses universitaires de France.

Dillon, P., Wang, R. et Tearle, P. (2007). Cultural disconnection in virtual education. Pedagogy, Culture \& Society, 15(2) 153174. https://doi.org/10.1080/14681360701403565

Fauconnier, G. et Turner, M. (2002). The way we think. Conceptual blending and the mind's hidden complexities. New York : Basic Books.

Fonseca, M. et Gajo, L. (2020). Le plurilinguisme dans les MOOCs: Profils d'usagers et fonctions du sous-titrage. Alsic. Apprentissage des Langues et Systèmes d'Information et de Communication, 23(2). https://doi.org/10.4000/alsic.4816

Fonseca, M. et Gajo, L. (2021). Le plurilinguisme dans les MOOCs : de nouvelles ressources pour la construction des savoirs. Bulletin suisse de linguistique appliquée, $\mathrm{n}^{\circ}$ spécial, 2, 165-181.

Gajo, L. (2007). Linguistic Knowledge and Subject Knowledge: How Does Bilingualism Contribute to Subject Developement? The International Journal of Bilingual Education and Bilingualism, 10(5), 563-581. https://doi.org/10.2167/beb460.0

Jacquinot, G. (2012). Image et pédagogie. PUF. (Ouvrage original publié en 1977)

Jaillet, A. (2014). Les films promoteurs de MOOC, une rhétorique de la « divisio ». Distances et médiations des savoirs. Distance and Mediation of Knowledge, 2(8). https://doi.org/10.4000/dms.951

Loizzo, J. et Ertmer, P. (2016). MOOCocracy: the learning culture of massive open online courses. Educational Technology Research and Development, 64(4), 1013-1032. https://doi.org/10.1007/s11423-016-9444-7

Kerr, R., Merciai, I. et Eradze, M. (2018). Addressing cultural and linguistic diversity in an online learning environment. Educational Media International, 55(4), 317-332. https://doi.org/10.1080/09523987.2018.1547546

Meunier, J.-P. et Peraya, D. (2010). Introduction aux théories de la communication. Bruxelles : De Boeck. (Ouvrage original publié en 1993)

Moeglin, P. (1998). L'industrialisation de la formation. État de la question. Documents, actes et rapports pour l'éducation. Centre national de documentation pédagogique. https://hal.archives-ouvertes.fr/hal-01387355

Nkuyubwatsi, B. (2014). Cultural Translation in Massive Open Online Courses (MOOCs). eLearning Papers, 37, 1-10. https://www.academia.edu/23880005/Cultural translation in MOOCspdf

Peltier, C. et Campion B. (2017). Regards pédagogique et communicationnel sur les capsules vidéo dans les MOOC. Distances et médiations des savoirs, 18. https://doi.org/10.4000/dms.1827

Peltier, C. et Campion, B. (2018). Constructions langagières, relation et cognition dans les capsules vidéo des MOOC. Pour une revisitation des possibilités éducatives de la vidéo. Distances et médiations des savoirs, 21. https://doi.org/10.4000/dms.2125

Phan, T. (2018). Instructional strategies that respond to global learners' needs in massive open online courses, Online learning, 22(2), 95-118. http://doi.org/10.24059/olj.v22i2.1160

Stratton, C. et Grace, R. (2016). Exploring Linguistic Diversity of MOOCs: Implications for International Development. Proceedings of the Association for Information Science and Technologie, 53(1). https://doi.org/10.1002/pra2.2016.14505301071

Young, J.R. (2012). The Object Formerly Known as the Textbook. The Chronicle of Higher Education, 27/01/2013. https://www.chronicle.com/article/the-object-formerly-known-as-the-textbook 